\title{
Conventional Versus Microwave Assisted Synthesis, Molecular Docking Studies and In Vitro Evaluation of Benzohydrazide Derivatives as New Acetylcholinesterase and Butyrylcholinesterase Inhibitors
}

Asad Ali' ${ }^{1 *}$, Anis Ur Rahman', Muhammad Ateeq ${ }^{1}$, Farhat Ullah², Uzma Rasheed Mughal ${ }^{3}$ and Abdul Wadood ${ }^{4}$

${ }^{1}$ Department of Chemistry, Abdul Wali Khan University, Mardan, Pakistan

${ }^{2}$ Department of Pharmacy, University of Malakand, Chakdara, Pakistan

${ }^{3} \mathrm{HEJ}$ Research Institute of Chemistry, International Center for Chemical and Biological Sciences, University of Karachi, Karachi, Pakistan

${ }^{4}$ Department of Biochemistry, Abdul Wali Khan University, Mardan, Pakistan

\begin{abstract}
In the course of studies directed towards the synthesis of novel AChE and BChE inhibitors, for the treatment of Alzheimer disease, we focused on the conventional versus microwave assisted synthesis of seventeen benzohydrazide derivatives and tested their ability as AChE and BChE inhibitors. These derivatives were characterized by FT-IR, ${ }^{1} \mathrm{H}-\mathrm{NMR},{ }^{13} \mathrm{C}-\mathrm{NMR}$ and EI-MS. Seventeen derivatives exhibited varied acetylcholinesterase inhibition with $\mathrm{IC}_{50}$ values ranging between $72.04 \pm 1.12$ to $1320.65 \pm 0.95 \mu \mathrm{M}$ as well as butyrylcholinesterase activity with $\mathrm{IC}_{50}$ values ranging between $3.04 \pm 1.48-1876.17 \pm 0.95 \mu \mathrm{M}$ as compared with standard eserine $\left(\mathrm{IC}_{50}=0.85 \pm 0.0001 \mu \mathrm{M}\right)$. Only two analogs $3 \mathrm{k}$ and 30 exhibited moderate acetylcholinesterase inhibitor potential with $I_{50}$ values $72.04 \pm 1.12$ and $94.06 \pm 1.17 \mu \mathrm{M}$ respectively. Five analogs $3 \mathrm{~d}, 3 \mathrm{~h}, 3 \mathrm{q}, 3 \mathrm{o}$ and $3 \mathrm{l}$ exhibited good potent butyrylcholinesterase inhibitory potential with $\mathrm{IC}_{50}$ values $3.04 \pm 1.48,9.01 \pm 0.58,15.12 \pm 0.66,45.00 \pm$ 0.99 and $50.19 \pm 0.62 \mu \mathrm{M}$ respectively. Molecular docking studies were carried out in order to find out the binding affinity of benzohydrazide derivatives with the enzyme.
\end{abstract}

Keywords: Benzohydrazide;Acetylcholinesterase; Butyrylcholinesterase; Microwave assisted; Docking studies

\section{Introduction}

Microwave irradiation has been effectively utilized as a nonconventional energy source in organic chemistry [1]. In conventional heating, energy is transferred from the surfaces of the material due to thermal gradients. However, microwave heating rather than heat transfer is the conversion of highly polarizing electromagnetic radiation to thermal energy which is delivered directly to materials through molecular interaction with the electromagnetic field. Better yields with higher product purities, shorter reaction times, milder reaction conditions and energy savings all have been reported. In fact, a number of reactions that do not occur by conventional heating can be accomplished using microwaves [2]. A great deal of literature has been found on the application of microwaves to heterocyclic chemistry [3], fullerene chemistry [4], cycloaddition reactions [5], polymers [6], homogeneous [7] and heterogeneous catalysis [8], green chemistry [9] and many more.

Alzheimer's disease (AD) is a complex neurodegenerative disorder which is irrevocable and is considered the most common cause of dementia which is a clinical syndrome that involves progressive deterioration of intellectual function [10]. Dementia can compromise several cognitive abilities including memory, reasoning, decision making, language and visuospatial. Daily activities of an individual like social activities, work and relationships are impaired by the cognitive and behavioral changes that follow dementia [11]. AD is also linked with hypertension and raised serum cholesterol [12]. AD is a complex disease, with no single cause, age is the major threat for the progress of $\mathrm{AD}$ [13]. It is estimated that in 2010 alone, $\mathrm{AD}$ has cost the world $\$ 604$ billion. Approximately 36 million case of AD worldwide today, is predicted to be tripled by 2050 [14]. Modern drug-discovery methodology aims on the synthesis of unique anti-Alzheimer agents which has the effectiveness of inhibiting both acetylcholinesterase and butyrylcholinesterase [15].

Acetylcholine (ACh) is an organic molecule that acts as a neurotransmitter and neuromodulator in the central nervous system.
ACh together with associated neurons in CNS forms a cholinergic system, which is inclined to initiate inhibitory actions and trigger cognitive loss associated with Alzheimer's disease [16,17]. Butyrylcholinesterase, another cholinesterase that catalyzes the hydrolysis acetylcholine. While AChE activity remains unchanged or declines, BChE activity gradually increases in patients with $\mathrm{AD}$ and, therefore, $\mathrm{BChE}$ is gaining interest [18]. It is reported that patients suffering from hyperlipidemia and hypertension have higher BChE enzymatic activity than those who experienced heart attack or undertaken medication with $\beta$-blockers. While inactivation of $\mathrm{AChE}$ in nerve synapses can be fatal, specific inhibition of BChE has no adverse effects [19]. Therefore, BChE is often taken as a fundamental supplement of novel drug development to handle with the problems relating Alzheimer disease.

A great deal of literature has been found on Schiff bases revealing their important biological activities and a wide range of significant practical applications. Some of their prominent biological activities include antibacterial [20], antifungal [21], antimalarial [22], antiviral [23], inter alia, antitumor [24], herbicidal [25], antioxidant [26], anticonvulsant [27], anti-inflammatory [28], antiproliferative [29], anti HIV [30], antiglycation [31] and lipid lowering [32] properties. Our research group is focused on development of lead molecules through green approach. Herein we are going to report benzohydrazide derivatives as new Acetylcholinesterase and butyrylcholinesterase inhibitors.

*Corresponding authors: Asad Ali, Department of Chemistry, Abdul Wali Khan University, Mardan-23200, Pakistan, Tel: 0092937929122; Fax: 0092937542188; E-mail: asad_org@yahoo.com

Received September 08, 2016; Accepted September 17, 2016; Published September 24, 2016

Citation: Ali A, Rahman AU, Ateeq M, Ullah F, Mughal UR, et al. (2016) Conventional Versus Microwave Assisted Synthesis, Molecular Docking Studies and In Vitro Evaluation of Benzohydrazide Derivatives as New Acetylcholinesterase and Butyrylcholinesterase Inhibitors. Med Chem (Los Angeles) 6: 583-592. doi:10.4172/2161-0444.1000403

Copyright: $\odot 2016$ Ali A, et al. This is an open-access article distributed under the terms of the Creative Commons Attribution License, which permits unrestricted use, distribution, and reproduction in any medium, provided the original author and source are credited. 
Citation: Ali A, Rahman AU, Ateeq M, Ullah F, Mughal UR, et al. (2016) Conventional Versus Microwave Assisted Synthesis, Molecular Docking Studies and In Vitro Evaluation of Benzohydrazide Derivatives as New Acetylcholinesterase and Butyrylcholinesterase Inhibitors. Med Chem (Los Angeles) 6: 583-592. doi:10.4172/2161-0444.1000403

\section{Experimental Section}

\section{General methods}

The progress of all reactions was monitored by TLC using pre coated silica gel aluminum plates (Kieselgel 60, 254) purchased from Merck (Germany). Chromatograms were visualized by UV at 254 and $365 \mathrm{~nm}$. Melting points of the synthesized compounds were determined in open capillaries using SMP10 melting point apparatus and are uncorrected. The FTIR spectra were performed on Schimadzu Fourier Transform Infra-Red Spectrophotometer model 270 using ATR (Attenuated total reflectance) facility. NMR spectra were recorded on Bruker Avance $300 \mathrm{MHz}$ and $500 \mathrm{MHz}$ spectrophotometers using DMSO as solvent. Electron ionization (EI) mass spectra (MS) were recorded on Jeol JMS-600H.

\section{General procedure for the synthesis of compounds}

Conventionally, Schiff bases 3a-3q were synthesized by condensing 2-aminobenzohydrazide and 4-methoxy benzo hydrazide via reflux with different substituted aromatic aldehydes in absolute ethanol containing acetic acid for 2-4 hours. After completion of reactions (TLC analysis), the reaction mixtures were cooled at ambient temperature, then ice cooled distilled water was added and allowed to withstand overnight. The precipitate formed were filtered, and dried up to afford the required compounds. The same reaction mixtures were also subjected to microwave irradiation for 2-6 minutes and the yield of reactions were noted. For structure confirmations, the synthesized products were investigated using ${ }^{1} \mathrm{H}$ NMR, ${ }^{13} \mathrm{C}$ NMR FTIR and EI-MS spectroscopy.

\section{Characterization of compounds}

(E) - 2 - a m ino- $N^{\prime}$ - (2-hydroxy - 5 - nit robenzylidene) benzohydrazide (3a): m.p. $274^{\circ} \mathrm{C}$; IR $\left(\overline{\mathbf{v}}, \mathbf{~ c m}^{-1}\right): 3465(\mathrm{OH}), 3354,3301$ $(\mathrm{NH}), 3217$ (imino $\mathrm{NH}), 3042\left(\mathrm{C}_{s p 2}-\mathrm{H}\right), 1661(\mathrm{C}=\mathrm{O}), 1608(\mathrm{C}=\mathrm{N}), 1519$ $(\mathrm{C}=\mathrm{C}), 1332(\mathrm{~N}-\mathrm{O}), 941(\mathrm{~N}-\mathrm{N}), 820,744\left(\right.$ Ar-H oop); ${ }^{1} \mathrm{H}$ NMR (300 MHz, DMSO- $\left.\boldsymbol{d}_{6}\right): \delta(\mathrm{ppm})=6.35\left(\mathrm{~s}, 2 \mathrm{H}, \mathrm{NH}_{2}\right), 6.57(\mathrm{t}, J=7.8 \mathrm{~Hz}, 1 \mathrm{H}$, $\left.\mathrm{H}_{\mathrm{Ar}}\right), 6.76\left(\mathrm{~d}, J=7.8 \mathrm{~Hz}, 1 \mathrm{H}, \mathrm{H}_{\mathrm{Ar}}\right), 7.10\left(\mathrm{~d}, J=9.0 \mathrm{~Hz}, 1 \mathrm{H}, \mathrm{H}_{\mathrm{Ar}}\right), 7.22(\mathrm{t}$, $\left.J=7.8 \mathrm{~Hz}, 1 \mathrm{H}, \mathrm{H}_{\mathrm{Ar}}\right), 7.60\left(\mathrm{~d}, J=7.8 \mathrm{~Hz}, 1 \mathrm{H}, \mathrm{H}_{\mathrm{Ar}}\right), 8.15(\mathrm{dd}, J=9.0 \mathrm{~Hz}$, $\left.J=2.7 \mathrm{~Hz}, 1 \mathrm{H}, \mathrm{H}_{\mathrm{Ar}}\right), 8.54\left(\mathrm{~m}, 1 \mathrm{H}, \mathrm{H}_{\mathrm{Ar}}\right), 8.66\left(\mathrm{~s}, 1 \mathrm{H}, \mathrm{H}_{\mathrm{vin}}\right), 12.05(\mathrm{~s}, 1 \mathrm{H}$, $\mathrm{NH}), 12.46$ (s (br), 1H, OH); ${ }^{13}$ C NMR (300 MHz, DMSO-d $) \delta 165.38$, $163.03,150.02,145.59,138.85,132.27,131.62,128.57,126.07,116.54$, 115.09, 120.11, 119.99, 112.83; EI-MS (EI, 70 eV): m/z $(\%)=300\left(\mathrm{M}^{+}\right.$, 19), 151 (04), 135 (05), 120 (100), 92 (18), 65 (09), 28 (18).

(E)-2-amino- $\mathrm{N}^{\prime}$ - (2-hydroxy-3-methoxybenzylidene) benzohydrazide (3b): m.p. $138^{\circ} \mathrm{C}$; IR $\left(\overline{\mathbf{v}}, \mathbf{c m}^{-1}\right): 3409(\mathrm{OH}), 3367$, $3316(\mathrm{NH}), 3204\left(\right.$ imino NH), $3066\left(\mathrm{C}_{s p 2}-\mathrm{H}\right), 2964,2883\left(\mathrm{C}_{s p 3}-\mathrm{H}\right), 1647$ $(\mathrm{C}=\mathrm{O}), 1604(\mathrm{C}=\mathrm{N}), 1505(\mathrm{C}=\mathrm{C}), 1251\left(\mathrm{C}_{s p 2}-\mathrm{O}\right), 1204\left(\mathrm{C}_{s p 3}-\mathrm{O}\right), 957(\mathrm{~N}-$ $\mathrm{N}), 845$ (Ar-H oop); ${ }^{1} \mathrm{H}$ NMR (300 MHz, DMSO-d $\left.\mathbf{~}_{6}\right): \delta$ (ppm) $=3.80$ $\left(\mathrm{s}, 3 \mathrm{H}, \mathrm{OCH}_{3}\right), 6.46\left(\mathrm{~s}, 2 \mathrm{H}, \mathrm{NH}_{2}\right), 6.58\left(\mathrm{~d}, J=7.8 \mathrm{~Hz}, 1 \mathrm{H}, \mathrm{H}_{\mathrm{Ar}}\right), 6.90(\mathrm{~d}$, $\left.J=8.1 \mathrm{~Hz}, 1 \mathrm{H}, \mathrm{H}_{\mathrm{Ar}}\right), 7.06\left(\mathrm{t}, J=7.8 \mathrm{~Hz}, 1 \mathrm{H}, \mathrm{H}_{\mathrm{Ar}}\right), 7.20(\mathrm{t}, J=8.1 \mathrm{~Hz}, 1 \mathrm{H}$, $\left.\mathrm{H}_{\mathrm{Ar}}\right), 7.28\left(\mathrm{t}, J=7.8 \mathrm{~Hz}, 1 \mathrm{H}, \mathrm{H}_{\mathrm{Ar}}\right), 7.58\left(\mathrm{~d}, J=7.8 \mathrm{~Hz}, 1 \mathrm{H}, \mathrm{H}_{\mathrm{Ar}}\right), 7.77(\mathrm{~d}$, $\left.J=8.1 \mathrm{~Hz}, 1 \mathrm{H}, \mathrm{H}_{\mathrm{Ar}}\right), 8.56\left(\mathrm{~s}, 1 \mathrm{H}, \mathrm{H}_{\mathrm{vin}}\right), 11.29(\mathrm{~s}, 1 \mathrm{H}, \mathrm{NH}), 11.82(\mathrm{~s}(\mathrm{br})$, $1 \mathrm{H}, \mathrm{OH}) ;{ }^{13} \mathrm{C}$ NMR (300 MHz, DMSO-d $) \delta 165.36,150.80,150.01$, 149.37, 146.27, 132.24, 131.97, 125.74, 119.76, 117.36, 116.65, 115.17, 114.05, 112.95, 56.37; EI-MS (EI, 70 eV): $m / z(\%)=285\left(\mathrm{M}^{+}, 18\right), 270$ (32), 259 (38), 254 (81), 241 (25), 197 (14), 161 (11), 136 (11), 120 (100), 92 (13), 65 (09), 28 (31).

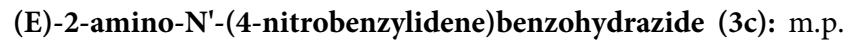
$243^{\circ} \mathrm{C}$; IR $\left(\overline{\mathbf{v}}, \mathbf{~ c m}^{-1}\right): 3333,3285(\mathrm{NH}), 3201($ imino NH$), 3039\left(\mathrm{C}_{s p 2}-\mathrm{H}\right)$, $1638(\mathrm{C}=\mathrm{O}), 1599(\mathrm{C}=\mathrm{N}), 1518(\mathrm{C}=\mathrm{C}), 1342(\mathrm{~N}-\mathrm{O}), 937(\mathrm{~N}-\mathrm{N}), 841$, 758 (Ar-H oop); ${ }^{1} \mathrm{H}$ NMR (300 MHz, DMSO-d $): \delta(\mathrm{ppm})=6.42(\mathrm{~s}$, $\left.2 \mathrm{H}, \mathrm{NH}_{2}\right), 6.57\left(\mathrm{t}, J=7.8 \mathrm{~Hz}, 1 \mathrm{H}, \mathrm{H}_{\mathrm{Ar}}\right), 6.76\left(\mathrm{~d}, J=8.10 \mathrm{~Hz}, 1 \mathrm{H}, \mathrm{H}_{\mathrm{Ar}}\right)$, $7.21\left(\mathrm{t}, J=7.8 \mathrm{~Hz}, 1 \mathrm{H}, \mathrm{H}_{\mathrm{Ar}}\right), 7.58\left(\mathrm{~d}, J=7.8 \mathrm{~Hz}, 1 \mathrm{H}, \mathrm{H}_{\mathrm{Ar}}\right), 7.95(\mathrm{~d}, J=$ $\left.8.7 \mathrm{~Hz}, 2 \mathrm{H}, \mathrm{H}_{\mathrm{Ar}}\right), 8.29\left(\mathrm{~d}, J=8.7 \mathrm{~Hz}, 2 \mathrm{H}, \mathrm{H}_{\mathrm{Ar}}\right), 8.47\left(\mathrm{~s}, 1 \mathrm{H}, \mathrm{H}_{\mathrm{vin}}\right), 11.88$ (s, 1H, NH); ${ }^{13} \mathrm{C}$ NMR (300 MHz, DMSO-d $) \delta 165.35,150.80,149.32$, $148.19,140.65,132.35,131.89,125.97,125.08,116.60,115.22,112.86$; EI-MS (EI, $70 \mathrm{eV}): m / z(\%)=284\left(\mathrm{M}^{+}, 09\right), 151(08), 135(02), 120$ (100), 92 (40), 77 (03), 65 (16), 28 (11).

(E)-2-amino- $\mathrm{N}^{\prime}$-(2,4-dichlorobenzylidene)benzohydrazide (3d): m.p. $209^{\circ} \mathrm{C}$; IR ( $\left.\overline{\mathbf{v}}, \mathbf{~ c m}^{-1}\right)$ : 3393, $3347(\mathrm{NH}), 3223$ (imino NH), 3042 $\left(\mathrm{C}_{s p 2}-\mathrm{H}\right), 1651(\mathrm{C}=\mathrm{O}), 1602(\mathrm{C}=\mathrm{N}), 1513(\mathrm{C}=\mathrm{C}), 1043(\mathrm{C}-\mathrm{Cl}), 944(\mathrm{~N}-$ $\mathrm{N}), 838,758$ (Ar-H oop); ${ }^{1} \mathrm{H}$ NMR (300 MHz, DMSO-d $\left.)_{6}\right): \delta(\mathrm{ppm})=$ $6.42\left(\mathrm{~s}, 2 \mathrm{H}, \mathrm{NH}_{2}\right), 6.56\left(\mathrm{t}, J=7.8 \mathrm{~Hz}, 1 \mathrm{H}, \mathrm{H}_{\mathrm{Ar}}\right), 6.75(\mathrm{~d}, J=8.1 \mathrm{~Hz}, 1 \mathrm{H}$, $\left.\mathrm{H}_{\mathrm{Ar}}\right), 7.20\left(\mathrm{t}, J=8.1 \mathrm{~Hz}, 1 \mathrm{H}, \mathrm{H}_{\mathrm{Ar}}\right), 7.51\left(\mathrm{dd}, J=8.4 \mathrm{~Hz}, 1.8 \mathrm{~Hz}, 1 \mathrm{H}, \mathrm{H}_{\mathrm{Ar}}\right)$, $7.58\left(\mathrm{~d}, J=7.8 \mathrm{~Hz}, 1 \mathrm{H}, \mathrm{H}_{\mathrm{Ar}}\right), 7.70\left(\mathrm{~d}, J=1.8 \mathrm{~Hz}, 1 \mathrm{H}, \mathrm{H}_{\mathrm{Ar}}\right), 7.99(\mathrm{~d}, J=8.4$ $\left.\mathrm{Hz}, 1 \mathrm{H}, \mathrm{H}_{\mathrm{Ar}}\right), 8.74\left(\mathrm{~s}, 1 \mathrm{H}, \mathrm{H}_{\mathrm{vin}}\right), 11.86(\mathrm{~s}, 1 \mathrm{H}, \mathrm{NH}) ;{ }^{13} \mathrm{C} \mathrm{NMR}(300 \mathrm{MHz}$, DMSO- $\left.\boldsymbol{d}_{6}\right) \delta 165.35,150.80,144.52,134.73,134.15,132.33,131.80$, 130.85, 129.17, 128.30, 127.32, 116.62, 115.12, 112.91; EI-MS (EI, 70 eV): $m / z(\%)=308\left(\mathrm{M}^{+}, 2\right),[\mathrm{M}-1]^{+} 307(04),[\mathrm{M}+1]^{+} 309(03), 214(18)$ 186 (21), 173 (27), 136 (20), 120 (100), 92 (31), 77 (20), 65 (13), 28 (25).

(E)-2-amino-N'-(4-methoxybenzylidene)benzohydrazide (3e): m.p. $234^{\circ} \mathrm{C}$; IR $\left(\overline{\mathbf{v}}, \mathbf{c m}^{-1}\right): 3325,3297(\mathrm{NH}), 3185$ (imino NH), 3058 $\left(\mathrm{C}_{s p 2}-\mathrm{H}\right), 2960,2905\left(\mathrm{C}_{s p 3}-\mathrm{H}\right), 1650(\mathrm{C}=\mathrm{O}), 1604(\mathrm{C}=\mathrm{N}), 1504(\mathrm{C}=\mathrm{C})$, $1360\left(\mathrm{CH}_{3}\right), 1246\left(\mathrm{C}_{s p 2}-\mathrm{O}\right), 1028\left(\mathrm{C}_{s p 3}-\mathrm{O}\right), 962(\mathrm{~N}-\mathrm{N}), 833,756(\mathrm{Ar}-\mathrm{H}$ oop); ${ }^{1} \mathrm{H}$ NMR (300 MHz, DMSO-d $\left.\boldsymbol{d}_{6}\right): \delta(\mathrm{ppm})=3.78\left(\mathrm{~s}, 3 \mathrm{H}, \mathrm{OCH}_{3}\right)$, $6.35\left(\mathrm{~s}, 2 \mathrm{H}, \mathrm{NH}_{2}\right), 6.71\left(\mathrm{t}, J=7.8 \mathrm{~Hz}, 1 \mathrm{H}, \mathrm{H}_{\mathrm{Ar}}\right), 6.76(\mathrm{~d}, J=7.8 \mathrm{~Hz}, 1 \mathrm{H}$, $\left.\mathrm{H}_{\mathrm{Ar}}\right), 6.86\left(\mathrm{t}, J=8.7 \mathrm{~Hz}, 2 \mathrm{H}, \mathrm{H}_{\mathrm{Ar}}\right), 6.98\left(\mathrm{~d}, J=8.7 \mathrm{~Hz}, 2 \mathrm{H}, \mathrm{H}_{\mathrm{Ar}}\right), 7.27(\mathrm{t}, J$ $\left.=7.8 \mathrm{~Hz}, 1 \mathrm{H}, \mathrm{H}_{\mathrm{Ar}}\right), 7.69\left(\mathrm{~d}, J=7.8 \mathrm{~Hz}, 1 \mathrm{H}, \mathrm{H}_{\mathrm{Ar}}\right), 8.75\left(\mathrm{~s}, 1 \mathrm{H}, \mathrm{H}_{\mathrm{vin} .}\right), 11.86$ (s, 1H, NH); ${ }^{13}$ C NMR (300 MHz, DMSO-d $)$ ) $\delta 165.35,162.05,150.80$, 146.79, 132.34, 131.89, 129.07, 127.52, 116.72, 115.27, 115.01, 112.79, 55.80. EI-MS (EI, $70 \mathrm{eV}): m / z(\%)=269\left(\mathrm{M}^{+}, 01\right), 210(14), 196(06)$, 136 (05), 130 (18), 120 (100), 92 (23), 65 (15), 51 (05), 28 (12).

(E)-2-amino-N'-(2,3,4-trimethoxybenzylidene)benzohydrazide (3f): m.p. $231{ }^{\circ} \mathrm{C}$; IR ( $\left.\overline{\mathbf{v}}, \mathbf{c m}^{-1}\right): 3375,3312(\mathrm{NH}), 3216$ (imino $\mathrm{NH}$ ), $3047\left(\mathrm{C}_{s p 2}-\mathrm{H}\right), 2970,2913\left(\mathrm{C}_{s p 3}-\mathrm{H}\right), 1632(\mathrm{C}=\mathrm{O}), 1597(\mathrm{C}=\mathrm{N}), 1502$ $(\mathrm{C}=\mathrm{C}), 1363\left(\mathrm{CH}_{3}\right), 1258\left(\mathrm{C}_{s p 2}-\mathrm{O}\right), 1036\left(\mathrm{C}_{s p 3}-\mathrm{O}\right), 945(\mathrm{~N}-\mathrm{N}), 837,752$ (Ar-H oop); ${ }^{1} \mathbf{H}$ NMR (300 MHz, DMSO-d $\left.{ }_{6}\right): \delta(\mathrm{ppm})=3.70(\mathrm{~s}, 3 \mathrm{H}$, $\left.\mathrm{OCH}_{3}\right), 3.82\left(\mathrm{~s}, 6 \mathrm{H}, \mathrm{OCH}_{3}\right), 6.32\left(\mathrm{~s}, 2 \mathrm{H}, \mathrm{NH}_{2}\right), 6.56(\mathrm{t}, J=7.5 \mathrm{~Hz}, 1 \mathrm{H}$, $\left.\mathrm{H}_{\mathrm{Ar}}\right), 6.74\left(\mathrm{~d}, J=8.1 \mathrm{~Hz}, 1 \mathrm{H}, \mathrm{H}_{\mathrm{Ar}}\right), 6.99\left(\mathrm{~s}, 2 \mathrm{H}, \mathrm{H}_{\mathrm{Ar}}\right), 7.19(\mathrm{t}, J=7.5 \mathrm{~Hz}$, $\left.1 \mathrm{H}, \mathrm{H}_{\mathrm{Ar}}\right), 7.54\left(\mathrm{~d}, J=7.5 \mathrm{~Hz}, 1 \mathrm{H}, \mathrm{H}_{\mathrm{Ar}}\right), 8.29\left(\mathrm{~s}, 1 \mathrm{H}, \mathrm{H}_{\mathrm{vin}}\right), 11.58(\mathrm{~s}, 1 \mathrm{H}$, $\mathrm{NH}$ ); ${ }^{13} \mathrm{C}$ NMR (300 MHz, DMSO-d $\left.\boldsymbol{d}_{6}\right) \delta$ (ppm) 165.42, 153.14, 149.92, $146.68,139.05,132.14,130.02,128.35,116.29,114.56,113.63,104.13$, 60.08, 55.90. EI-MS (EI, $70 \mathrm{eV}): m / z(\%)=329\left(\mathrm{M}^{+}, 68\right), 195(05), 136$ (12), 120 (100), 92 (13), 65 (05), 28 (01).

(E)-2-amino- $\mathrm{N}^{\prime}$-(3,4-dimethoxybenzylidene)benzohydrazide (3g): m.p. $125^{\circ} \mathrm{C}$; IR $\left(\overline{\mathbf{v}}, \mathbf{c m}^{-1}\right): 3371,3320(\mathrm{NH}), 3221$ (imino $\mathrm{NH}$ ), $3049\left(\mathrm{C}_{s p 2}-\mathrm{H}\right), 2973,2922\left(\mathrm{C}_{s p 3}-\mathrm{H}\right), 1640(\mathrm{C}=\mathrm{O}), 1601(\mathrm{C}=\mathrm{N}), 1500$ $(\mathrm{C}=\mathrm{C}), 1255\left(\mathrm{C}_{s p 2}-\mathrm{O}\right), 1033\left(\mathrm{C}_{s p 3}-\mathrm{O}\right), 945(\mathrm{~N}-\mathrm{N}), 825,755(\mathrm{Ar}-\mathrm{H}$ oop); ${ }^{1}$ H NMR (300 MHz, DMSO-d d $\left._{6}\right): \delta(\mathrm{ppm})=3.67\left(\mathrm{~s}, 3 \mathrm{H}, \mathrm{OCH}_{3}\right), 3.78$ $\left(\mathrm{s}, 3 \mathrm{H}, \mathrm{OCH}_{3}\right), 6.35\left(\mathrm{~s}, 2 \mathrm{H}, \mathrm{NH}_{2}\right), 6.76\left(\mathrm{t}, J=7.8 \mathrm{~Hz}, 1 \mathrm{H}, \mathrm{H}_{\mathrm{Ar}}\right), 6.71$ $\left(\mathrm{d}, J=7.8 \mathrm{~Hz}, 1 \mathrm{H}, \mathrm{H}_{\mathrm{Ar}}\right), 6.86\left(\mathrm{~d}, J=8.5 \mathrm{~Hz}, 1 \mathrm{H}, \mathrm{H}_{\mathrm{Ar}}\right), 6.98(\mathrm{~d}, J=8.5$ $\left.\mathrm{Hz}, 1 \mathrm{H}, \mathrm{H}_{\mathrm{Ar}}\right), 7.28\left(\mathrm{t}, J=7.8 \mathrm{~Hz}, 1 \mathrm{H}, \mathrm{H}_{\mathrm{Ar}}\right), 7.60(\mathrm{~d}, J=7.8 \mathrm{~Hz}, 1 \mathrm{H}$, $\left.\mathrm{H}_{\mathrm{Ar}}\right), 7.66\left(\mathrm{~s}, 1 \mathrm{H}, \mathrm{H}_{\mathrm{Ar}}\right), 8.56\left(\mathrm{~s}, 1 \mathrm{H}, \mathrm{H}_{\mathrm{vin}}\right), 11.29(\mathrm{~s}, 1 \mathrm{H}, \mathrm{NH}) ;{ }^{13} \mathrm{C} \mathrm{NMR}$ (300 MHz, DMSO-d $) \delta 165.35,150.80,150.06,148.90,145.57,132.53$, $131.89,127.38,126.29,116.86,115.20,112.76,110.20,109.87,55.98$; EIMS (EI, $70 \mathrm{eV}): m / z(\%)=299\left(\mathrm{M}^{+}, 2\right), 253(64), 238(100), 196(03)$, 147 (26), 135 (09), 119 (14), 105 (07), 92 (04), 77 (06), 65 (02), 51 (02), 28 (04).

(E)-2-amino-N'-(4-hydroxybenzylidene)benzohydrazide (3h): m.p. $218{ }^{\circ} \mathrm{C}$; IR ( $\left(\overline{\mathbf{v}}, \mathbf{c m}^{-1}\right): 3425(\mathrm{OH}), 3355,3314(\mathrm{NH}), 3226$ (imino $\mathrm{NH}), 3056\left(\mathrm{C}_{s p 2}-\mathrm{H}\right), 1649(\mathrm{C}=\mathrm{O}), 1602(\mathrm{C}=\mathrm{N}), 1511(\mathrm{C}=\mathrm{C}), 953(\mathrm{~N}-\mathrm{N})$, 
Citation: Ali A, Rahman AU, Ateeq M, Ullah F, Mughal UR, et al. (2016) Conventional Versus Microwave Assisted Synthesis, Molecular Docking Studies and In Vitro Evaluation of Benzohydrazide Derivatives as New Acetylcholinesterase and Butyrylcholinesterase Inhibitors. Med Chem (Los Angeles) 6: 583-592. doi:10.4172/2161-0444.1000403

844, 756 (Ar-H oop); ${ }^{1} \mathbf{H}$ NMR (500 MHz, DMSO- $\left.\boldsymbol{d}_{6}\right): \delta(\mathrm{ppm})=6.13$ $\left(\mathrm{s}, 2 \mathrm{H}, \mathrm{NH}_{2}\right), 6.70\left(\mathrm{~d}, J=7.8 \mathrm{~Hz}, 1 \mathrm{H}, \mathrm{H}_{\mathrm{Ar}}\right), 6.76\left(\mathrm{t}, J=7.8 \mathrm{~Hz}, 1 \mathrm{H}, \mathrm{H}_{\mathrm{Ar}}\right)$, $6.86\left(\mathrm{~d}, J=8.5 \mathrm{~Hz}, 2 \mathrm{H}, \mathrm{H}_{\mathrm{Ar}}\right), 7.21\left(\mathrm{t}, J=7.8 \mathrm{~Hz}, 1 \mathrm{H}, \mathrm{H}_{\mathrm{Ar}}\right), 7.54(\mathrm{~d}, J=$ $\left.8.5 \mathrm{~Hz}, 2 \mathrm{H}, \mathrm{H}_{\mathrm{Ar}}\right), 7.65\left(\mathrm{~d}, J=7.8 \mathrm{~Hz}, 1 \mathrm{H}, \mathrm{H}_{\mathrm{Ar}}\right), 8.45\left(\mathrm{~s}, 1 \mathrm{H}, \mathrm{H}_{\mathrm{vin}}\right), 9.95$ (s (br), 1H, OH), 11.65 (s, 1H, NH); ${ }^{13}$ C NMR (300 MHz, DMSO- $\left.d_{6}\right)$ $\delta 165.35,153.82,150.79,148.79,133.11,132.53,131.86,127.78,116.84$, 115.24, 113.02, 112.60; EI-MS (EI, 70 eV): $m / z(\%)=255\left(\mathrm{M}^{+}, 03\right), 195$ (04), 167 (07), 147 (25), 135 (06), 120 (100), 92 (14), 65 (07), 28 (13).

(E)-2-amino-N'-(2-methylbenzylidene)benzohydrazide

(3i):

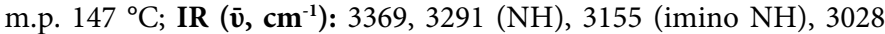
$\left(\mathrm{C}_{s p 2}-\mathrm{H}\right), 2956,2920\left(\mathrm{C}_{s p 3}-\mathrm{H}\right), 1643(\mathrm{C}=\mathrm{O}), 1606(\mathrm{C}=\mathrm{N}), 1514(\mathrm{C}=\mathrm{C})$, $948(\mathrm{~N}-\mathrm{N}), 844,743$ (Ar-H oop); ${ }^{1} \mathrm{H}$ NMR (300 MHz, DMSO-d $\left.)_{6}\right): \delta$ $(\mathrm{ppm})=2.42\left(\mathrm{~s}, 3 \mathrm{H}, \mathrm{CH}_{3}\right), 6.38\left(\mathrm{~s}, 2 \mathrm{H}, \mathrm{NH}_{2}\right), 6.65(\mathrm{~d}, J=7.8 \mathrm{~Hz}, 1 \mathrm{H}$, $\left.\mathrm{H}_{\mathrm{Ar}}\right), 6.76\left(\mathrm{t}, J=7.8 \mathrm{~Hz}, 1 \mathrm{H}, \mathrm{H}_{\mathrm{Ar}}\right), 7.13-7.32\left(\mathrm{~m}, 4 \mathrm{H}, \mathrm{H}_{\mathrm{Ar}}\right), 7.56(\mathrm{~d}, J=$ $\left.7.8 \mathrm{~Hz}, 1 \mathrm{H}, \mathrm{H}_{\mathrm{Ar}}\right), 7.81\left(\mathrm{~d}, J=8.4 \mathrm{~Hz}, 1 \mathrm{H}, \mathrm{H}_{\mathrm{Ar}}\right), 8.67\left(\mathrm{~s}, 1 \mathrm{H}, \mathrm{H}_{\mathrm{vin}}\right), 11.55$ $(\mathrm{s}, 1 \mathrm{H}, \mathrm{NH}) ;{ }^{13} \mathrm{C}$ NMR (300 MHz, DMSO-d $) \delta 165.35,150.78,146.37$, $137.00,132.53,131.89,131.80,131.55,129.31,128.99,127.03,116.86$, 115.20, 112.59, 19.55; EI-MS (EI, 70 eV): $m / z(\%)=253\left(\mathrm{M}^{+}, 69\right), 165$ (04), 147 (56), 136 (20), 120 (100), 92 (28), 77 (07) 65 (13), 28 (03).

( E ) - N ' - ( 2 - h y d roxy - 5 - n i trob enzyliden e ) - 4 methoxybenzohydrazide (3j): m.p. $281^{\circ} \mathrm{C}$; IR $\left(\overline{\mathbf{v}}, \mathbf{c m}^{-1}\right): 3385(\mathrm{OH})$,

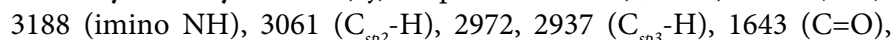
$1605(\mathrm{C}=\mathrm{N}), 1506(\mathrm{C}=\mathrm{C}), 1338(\mathrm{NO}), 1257\left(\mathrm{C}_{s p 2}-\mathrm{O}\right), 1026\left(\mathrm{C}_{s p p}-\mathrm{O}\right), 958$ $(\mathrm{N}-\mathrm{N}), 843$ (Ar-H oop); ${ }^{1} \mathrm{H}$ NMR (500 MHz, DMSO-d $): \delta(\mathrm{ppm})=$ $3.83\left(\mathrm{~s}, 3 \mathrm{H}, \mathrm{OCH}_{3}\right), 7.06\left(\mathrm{~d}, J=8.5 \mathrm{~Hz}, 2 \mathrm{H}, \mathrm{H}_{\mathrm{Ar}}\right), 7.10(\mathrm{~d}, J=9.0 \mathrm{~Hz}, 1 \mathrm{H}$, $\left.\mathrm{H}_{\mathrm{Ar}}\right), 7.93\left(\mathrm{~d}, J=8.5 \mathrm{~Hz}, 2 \mathrm{H}, \mathrm{H}_{\mathrm{Ar}}\right), 8.16\left(\mathrm{dd}, J=9.0 \mathrm{~Hz}, 3.0 \mathrm{~Hz}, 1 \mathrm{H}, \mathrm{H}_{\mathrm{Ar}}\right)$, $8.56\left(\mathrm{~s}, 1 \mathrm{H}, \mathrm{H}_{\mathrm{s}}\right), 8.71\left(\mathrm{~s}, 1 \mathrm{H}, \mathrm{H}_{\mathrm{vin}}\right), 12.15(\mathrm{~s}, 1 \mathrm{H}, \mathrm{NH}), 12.39(\mathrm{~s}(\mathrm{br}), 1 \mathrm{H}$, $\mathrm{OH}) ;{ }^{13} \mathrm{C}$ NMR (300 MHz, DMSO-d $) \delta 163.46,162.13,160.99,146.45$, $137.55,129.68,128.69,126.24,124.74,120.40,121.03,113.70,55.35$; EIMS (EI, $70 \mathrm{eV}): m / z(\%)=315\left(\mathrm{M}^{+}, 10\right), 285(02), 165(01), 151(14)$, 135 (100), 119 (01), 107 (10), 92 (09), 77 (13), 64 (03), 28 (04).

(E) - N' - ( 2 - hydroxy - 3 - methoxybenzylidene ) - 4 methoxybenzohydrazide (3k): m.p. $117^{\circ} \mathrm{C}$; IR $\left(\overline{\mathbf{v}}, \mathbf{c m}^{-1}\right): 3466(\mathrm{OH})$, 3189 (imino $\mathrm{NH}), 3059\left(\mathrm{C}_{s p 2}-\mathrm{H}\right), 2929,2877\left(\mathrm{C}_{s p 3}-\mathrm{H}\right), 1651(\mathrm{C}=\mathrm{O})$, $1606(\mathrm{C}=\mathrm{N}), 1512(\mathrm{C}=\mathrm{C}), 1250\left(\mathrm{C}_{s p 2}-\mathrm{O}\right), 1034\left(\mathrm{C}_{s p 3}-\mathrm{O}\right), 958(\mathrm{~N}-\mathrm{N}), 849$, 783 (Ar-H oop); ${ }^{1} \mathbf{H}$ NMR (500 MHz, DMSO-d $\left.{ }_{6}\right): \delta(\mathrm{ppm})=3.80(\mathrm{~s}$, $\left.3 \mathrm{H}, \mathrm{OCH}_{3}\right), 3.83\left(\mathrm{~s}, 3 \mathrm{H}, \mathrm{OCH}_{3}\right), 6.85\left(\mathrm{t}, J=8.0 \mathrm{~Hz}, 1 \mathrm{H}, \mathrm{H}_{\mathrm{Ar}}\right), 7.02(\mathrm{~d}$, $\left.J=8.0 \mathrm{~Hz}, 1 \mathrm{H}, \mathrm{H}_{\mathrm{Ar}}\right), 7.07\left(\mathrm{~d}, J=8.5 \mathrm{~Hz}, 2 \mathrm{H}, \mathrm{H}_{\mathrm{Ar}}\right), 7.11(\mathrm{~d}, J=8.0 \mathrm{~Hz}$, $\left.1 \mathrm{H}, \mathrm{H}_{\mathrm{Ar}}\right), 7.92\left(\mathrm{t}, J=8.5 \mathrm{~Hz}, 2 \mathrm{H}, \mathrm{H}_{\mathrm{Ar}}\right), 8.62\left(\mathrm{~s}, 1 \mathrm{H}, \mathrm{H}_{\mathrm{vin}}\right), 11.04(\mathrm{~s}, 1 \mathrm{H}$, $\mathrm{NH}), 11.95$ (s (br), $1 \mathrm{H}, \mathrm{OH})$; ${ }^{13} \mathrm{C}$ NMR (300 MHz, DMSO-d $) \delta 163.32$, $162.08,152.88,146.54,129.66,126.29,124.74,119.81,117.93,113.72$, 112.90, 56.54, 55.41; EI-MS (EI, $70 \mathrm{eV}): m / z(\%)=300\left(\mathrm{M}^{+}, 77\right), 177$ (08), 151 (24), 135 (100), 92 (06), 77 (07), 65 (04), 28 (29).

(E)-4-methoxy-N'-(4-nitrobenzylidene)benzohydrazide (31): m.p. $212^{\circ} \mathrm{C}$; IR ( $\left.\overline{\mathbf{v}}, \mathbf{c m}^{-1}\right)$ : 3110 (imino NH), $3065\left(\mathrm{C}_{s p 2}-\mathrm{H}\right), 2945,2863$ $\left(\mathrm{C}_{s p \mathrm{p}}-\mathrm{H}\right), 1649(\mathrm{C}=\mathrm{O}), 1600(\mathrm{C}=\mathrm{N}), 1506(\mathrm{C}=\mathrm{C}), 1246\left(\mathrm{C}_{s p 2}-\mathrm{O}\right), 1024$ $\left(\mathrm{C}_{s p 3}-\mathrm{O}\right), 944(\mathrm{~N}-\mathrm{N}), 839$ (Ar-H oop); ${ }^{1}$ H NMR (500 MHz, DMSO-d $)$ : $\delta(\mathrm{ppm})=3.83\left(\mathrm{~s}, 3 \mathrm{H}, \mathrm{OCH}_{3}\right), 7.07\left(\mathrm{~d}, J=8.5 \mathrm{~Hz}, 2 \mathrm{H}, \mathrm{H}_{\mathrm{Ar}}\right), 7.93(\mathrm{~d}, J$ $\left.=8.5 \mathrm{~Hz}, 2 \mathrm{H}, \mathrm{H}_{\mathrm{As}}\right), 7.97\left(\mathrm{~d}, J=8.5 \mathrm{~Hz}, 2 \mathrm{H}, \mathrm{H}_{\mathrm{Ar}}\right), 8.29(\mathrm{dr}, J=8.5 \mathrm{~Hz}$, $\left.2 \mathrm{H}, \mathrm{H}_{\mathrm{Ar}}\right), 8.53\left(\mathrm{~s}, 1 \mathrm{H}, \mathrm{H}_{\text {vin }}\right), 12.03(\mathrm{~s}, 1 \mathrm{H}, \mathrm{NH}) ;{ }^{13} \mathrm{C}$ NMR $(300 \mathrm{MHz}$, DMSO-d6) $\delta 163.37,162.11,148.79,146.43,140.72,129.69,125.92$, 125.58, 124.74, 113.69, 55.42; EI-MS (EI, $70 \mathrm{eV}): \mathrm{m} / z(\%)=299\left(\mathrm{M}^{+}\right.$, 24), 251 (04), 176 (14), 151 (47), 135 (100), 107 (25).

(E)-N'-(2,4-dichlorobenzylidene)-4-methoxybenzohydrazide (3m): m.p. $130^{\circ} \mathrm{C}$; IR ( $\left.\mathbf{v}, \mathbf{~ c m}^{-1}\right)$ : 3157 (imino NH), $3062\left(\mathrm{C}_{\text {sp }}-\mathrm{H}\right), 2949$, $2861\left(\mathrm{C}_{s p 3}-\mathrm{H}\right), 1634(\mathrm{C}=\mathrm{O}), 1604(\mathrm{C}=\mathrm{N}), 1506(\mathrm{C}=\mathrm{C}), 1256\left(\mathrm{C}_{s p 2}-\mathrm{O}\right)$, 1047 (C $\left.{ }_{s p 3}-\mathrm{O}, \mathrm{C}-\mathrm{Cl}\right), 954$ (N-N), 847 (Ar-H oop); ${ }^{1} \mathrm{H}$ NMR $(500 \mathrm{MHz}$, DMSO- $\left.\boldsymbol{d}_{6}\right): \delta(\mathrm{ppm})=3.83\left(\mathrm{~s}, 3 \mathrm{H}, \mathrm{OCH}_{3}\right), 7.06(\mathrm{~d}, J=8.5 \mathrm{~Hz}, 2 \mathrm{H}$, $\left.\mathrm{H}_{\mathrm{Ar}}\right), 7.51\left(\mathrm{~d}, J=8.5 \mathrm{~Hz}, 1 \mathrm{H}, \mathrm{H}_{\mathrm{Ar}}\right), 7.70\left(\mathrm{~m}, 1 \mathrm{H}, \mathrm{H}_{\mathrm{Ar}}\right), 7.92(\mathrm{~d}, J=8.5$
$\left.\mathrm{Hz}, 2 \mathrm{H}, \mathrm{H}_{\mathrm{Ar}}\right), 8.02\left(\mathrm{~d}, J=8.5 \mathrm{~Hz}, 1 \mathrm{H}, \mathrm{H}_{\mathrm{Ar}}\right), 8.79\left(\mathrm{~s}, 1 \mathrm{H}, \mathrm{H}_{\mathrm{vin}}\right), 11.98(\mathrm{~s}$, $1 \mathrm{H}, \mathrm{NH}) ;{ }^{13} \mathrm{C}$ NMR (300 MHz, DMSO-d $) \delta 162.21,161.81,144.54$, $134.80,134.10,130.96,129.68,129.00,128.30,127.40,124.68,113.70$, 55.30; EI-MS (EI, $70 \mathrm{eV}): m / z(\%)=323\left(\mathrm{M}^{+}, 01\right), 171(02), 151(19)$, 135 (100), 123 (02), 107 (05), 92 (05), 77 (05), 64 (02), 44 (02), 21 (06).

(E)-4-methoxy-N'-(4-methoxybenzylidene)benzohydrazide (3n): m.p. $172{ }^{\circ} \mathrm{C}$; IR ( $\left.\overline{\mathbf{v}}, \mathbf{c m}^{-1}\right)$ : $3203\left(\right.$ imino NH), $3065\left(\mathrm{C}_{s p 2}-\mathrm{H}\right), 2965$, $2889\left(\mathrm{C}_{s p 3}-\mathrm{H}\right), 1649(\mathrm{C}=\mathrm{O}), 1605(\mathrm{C}=\mathrm{N}), 1504(\mathrm{C}=\mathrm{C}), 1249\left(\mathrm{C}_{s p 2}-\mathrm{O}\right)$, $1024\left(\mathrm{C}_{s p 3}-\mathrm{O}\right), 955$ (N-N), 842 (Ar-H oop); ${ }^{1} \mathbf{H}$ NMR (300 $\mathbf{~ M H z}$, DMSO- $\left.\boldsymbol{d}_{6}\right): \delta(\mathrm{ppm})=3.80\left(\mathrm{~s}, 3 \mathrm{H}, \mathrm{OCH}_{3}\right), 3.82\left(\mathrm{~s}, 3 \mathrm{H}, \mathrm{OCH}_{3}\right), 7.00$ - $7.06\left(\mathrm{~m}, 4 \mathrm{H}, \mathrm{H}_{\mathrm{Ar}}\right), 7.65\left(\mathrm{~d}, J=8.5 \mathrm{~Hz}, 2 \mathrm{H}, \mathrm{H}_{\mathrm{Ar}}\right), 7.89(\mathrm{~d}, J=8.5 \mathrm{~Hz}$, $\left.2 \mathrm{H}, \mathrm{H}_{\mathrm{Ar}}\right), 8.38\left(\mathrm{~s}, 1 \mathrm{H}, \mathrm{H}_{\text {vin }}\right), 11.57(\mathrm{~s}, 1 \mathrm{H}, \mathrm{NH}) ;{ }^{13} \mathrm{C}$ NMR $(300 \mathrm{MHz}$, DMSO-d $d_{6} \delta 162.57,161.92,161.15,147.79,129.68,128.60,127.81$, 124.69, 115.16, 113.66, 55.42; EI-MS (EI, $70 \mathrm{eV}): \mathrm{m} / z(\%)=284\left(\mathrm{M}^{+}\right.$, 18), 268 (32), 240 (05), 161 (26), 151 (86), 135 (100), 107 (14), 92 (12), 77 (20), 64 (04).

(E)-4-methoxy-N'-(4-(methylthio)benzylidene)benzohydrazide (3o): m.p. $187^{\circ} \mathrm{C}$; IR ( $\left.\overline{\mathbf{v}}, \mathbf{c m}^{-1}\right): 3122$ (imino $\left.\mathrm{NH}\right), 3064\left(\mathrm{C}_{s p 2}-\mathrm{H}\right)$, 2959, $2873\left(\mathrm{C}_{s p 3}-\mathrm{H}\right), 1655(\mathrm{C}=\mathrm{O}), 1605(\mathrm{C}=\mathrm{N}), 1507(\mathrm{C}=\mathrm{C}), 1308$ (S$\left.\mathrm{CH}_{3}\right), 1259\left(\mathrm{C}_{s p 2}-\mathrm{O}\right), 1025\left(\mathrm{C}_{s p 3}-\mathrm{O}\right), 960(\mathrm{~N}-\mathrm{N}) ;{ }^{1} \mathrm{H}$ NMR $(500 \mathrm{MHz}$, DMSO- $\left.\boldsymbol{d}_{6}\right): \delta(\mathrm{ppm})=2.51\left(\mathrm{~s}, 3 \mathrm{H}, \mathrm{SCH}_{3}\right), 3.82\left(\mathrm{~s}, 3 \mathrm{H}, \mathrm{OCH}_{3}\right), 7.04(\mathrm{~d}$, $\left.J=8.5 \mathrm{~Hz}, 2 \mathrm{H}, \mathrm{H}_{\mathrm{Ar}}\right), 7.32\left(\mathrm{~d}, J=8.0 \mathrm{~Hz}, 2 \mathrm{H}, \mathrm{H}_{\mathrm{Ar}}\right), 7.64(\mathrm{~d}, J=7.5 \mathrm{~Hz}$, $\left.2 \mathrm{H}, \mathrm{H}_{\mathrm{Ar}}\right), 7.89\left(\mathrm{~d}, J=8.5 \mathrm{~Hz}, 2 \mathrm{H}, \mathrm{H}_{\mathrm{Ar}}\right), 8.38\left(\mathrm{~s}, 1 \mathrm{H}, \mathrm{H}_{\mathrm{vin}}\right), 11.67(\mathrm{~s}, 1 \mathrm{H}$, $\mathrm{NH}) ;{ }^{\mathrm{Ar}} \mathrm{C}$ NMR (300 MHz, DMSO-d $\left.{ }_{6}\right) \delta 162.41,161.81,147.75,139.30$, 133.09, 129.68, 128.35, 126.71, 124.74, 113.73, 55.35, 15.72; EI-MS (EI, $70 \mathrm{eV}): m / z(\%)=300\left(\mathrm{M}^{+}, 47\right), 177$ (9), 151 (49), 135 (100), 107 (07), $92(8), 77$ (12), 64 (2).

(E)-4-methoxy-N'-(4-methylbenzylidene)benzohydrazide (3p): m.p. $184^{\circ} \mathrm{C}$; IR $\left(\overline{\mathbf{v}}, \mathbf{c m}^{-1}\right): 3156$ (imino NH), $3062\left(\mathrm{C}_{s p 2}-\mathrm{H}\right), 2924,2850$ $\left(\mathrm{C}_{s p 3}-\mathrm{H}\right), 1659(\mathrm{C}=\mathrm{O}), 1605(\mathrm{C}=\mathrm{N}), 1508(\mathrm{C}=\mathrm{C}), 1365\left(\mathrm{CH}_{3}\right), 1250$ $\left(\mathrm{C}_{s p 2}^{s p}-\mathrm{O}\right), 1026\left(\mathrm{C}_{s p 3}-\mathrm{O}\right), 940(\mathrm{~N}-\mathrm{N}), 838$ (Ar-H oop); ${ }^{1} \mathrm{H}$ NMR (500 MHz, DMSO-d $\left.\boldsymbol{d}_{6}\right): \delta(\mathrm{ppm})=2.36\left(\mathrm{~s}, 3 \mathrm{H}, \mathrm{CH}_{3}\right), 3.82\left(\mathrm{~s}, 3 \mathrm{H}, \mathrm{OCH}_{3}\right), 7.05$ $\left(\mathrm{d}, J=8.5 \mathrm{~Hz}, 2 \mathrm{H}, \mathrm{H}_{\mathrm{Ar}}\right), 7.26\left(\mathrm{~d}, J=7.5 \mathrm{~Hz}, 2 \mathrm{H}, \mathrm{H}_{\mathrm{Ar}}\right), 7.60(\mathrm{~d}, J=7.5 \mathrm{~Hz}$ $\left.2 \mathrm{H}, \mathrm{H}_{\mathrm{Ar}}\right), 7.89\left(\mathrm{~d}, J=8.5 \mathrm{~Hz}, 2 \mathrm{H}, \mathrm{H}_{\mathrm{Ar}}\right), 8.39\left(\mathrm{~s}, 1 \mathrm{H}, \mathrm{H}_{\text {vin }}\right), 11.65(\mathrm{~s}, 1 \mathrm{H}$, $\mathrm{NH}) ;{ }^{\mathrm{Ar}} \mathrm{C}$ NMR (300 MHz, DMSO-d $\left.{ }_{6}\right) \delta 162.21,161.78,148.00,143.02$, 131.85, 129.68, 129.27, 126.37, 124.74, 113.73, 55.35, 21.67; EI-MS (EI, $70 \mathrm{eV}): m / z(\%)=268\left(\mathrm{M}^{+}, 4\right), 151(30), 135(100), 107(4), 92(4), 77$ (06), 64 (2), 28 (2)

N',N'"-((1E,1'E)-1,4-phenylenebis(methanylylidene))bis(2aminobenzohydrazide) (3q): m.p. $253^{\circ} \mathrm{C}$; IR $\left(\overline{\mathbf{v}}, \mathbf{c m}^{-1}\right): 3350,3317$ $(\mathrm{NH}), 3205$ (imino $\mathrm{NH}), 3057\left(\mathrm{C}_{s p 2}-\mathrm{H}\right), 1634(\mathrm{C}=\mathrm{O}), 1599(\mathrm{C}=\mathrm{N})$, $1514(\mathrm{C}=\mathrm{C}), 937(\mathrm{~N}-\mathrm{N}), 842,748$ (Ar-H oop); ${ }^{1} \mathrm{H}$ NMR $(300 \mathrm{MHz}$, DMSO- $\left.\boldsymbol{d}_{6}\right): \delta(\mathrm{ppm})=5.78\left(\mathrm{~s}, 4 \mathrm{H}, \mathrm{NH}_{2}\right), 6.62-6.67\left(\mathrm{~m}, 4 \mathrm{H}, \mathrm{H}_{\mathrm{Ar}}\right), 7.18$ $\left(\mathrm{t}, J=7.8 \mathrm{~Hz}, 2 \mathrm{H}, \mathrm{H}_{\mathrm{Ar}}\right), 7.29\left(\mathrm{~s}, 4 \mathrm{H}, \mathrm{H}_{\mathrm{Ar}}\right), 7.61\left(\mathrm{~d}, J=7.8 \mathrm{~Hz}, 2 \mathrm{H}, \mathrm{H}_{\mathrm{Ar}}\right)$, $8.38\left(\mathrm{~s}, 2 \mathrm{H}, \mathrm{H}_{\mathrm{vin}}\right), 11.88(\mathrm{~s}, 2 \mathrm{H}, \mathrm{NH}) ;{ }^{13} \mathrm{C}$ NMR (300 MHz, DMSO-d $\left.\boldsymbol{d}_{6}\right)$ $\delta 165.35,150.80,148.79,133.30,132.46,131.89,126.48,116.86,115.24$ 112.65; EI-MS (EI, $70 \mathrm{eV}): m / z(\%)=400\left(\mathrm{M}^{+}, 57\right), 180(08), 162(09)$, 120 (100), 92 (32), 77 (19), 65 (16), 50 (06), 28 (18).

\section{Results and Discussion}

\section{Chemistry}

The Schiff base ligands 3a-3q were synthesized using conventional method as well as microwave irradiations. Conventionally, 2-aminobenzohydrazide and 4-methoxy benzohydrazide were refluxed with substituted benzaldehydes (Table 1) in ethanol as a solvent and acetic acid as a catalyst. In an experimental procedure, a mixture of 2-aminobenzohydrazide/4-methoxybenzohydrazide $(1 \mathrm{mmol})$, substituted benzaldehydes $(1 \mathrm{mmol})$ and $2-3$ drops of acetic acid in 
Citation: Ali A, Rahman AU, Ateeq M, Ullah F, Mughal UR, et al. (2016) Conventional Versus Microwave Assisted Synthesis, Molecular Docking Studies and In Vitro Evaluation of Benzohydrazide Derivatives as New Acetylcholinesterase and Butyrylcholinesterase Inhibitors. Med Chem (Los Angeles) 6: 583-592. doi:10.4172/2161-0444.1000403

absolute ethanol $(15 \mathrm{ml})$ were refluxed for $2-4$ hours (Scheme 1 ). These reaction mixtures were also subjected to microwave irradiation for 2-5 minutes and the yield of reactions were noted. Comparison between conventional and MW assisted synthesis is given in Table 2. The Progress of reaction was monitored by TLC. In either method, on completion of reaction, the mixture was cooled and the solvent was evaporated under reduced pressure to obtain the desired products which were then washed with DCM $(25 \mathrm{ml})$ and dried. The structures were determined by using spectroscopic techniques such as FTIR, ${ }^{1} \mathrm{HNMR},{ }^{13} \mathrm{C}$ NMR and EI-MS spectroscopy.

\section{Acetyl cholinesterase and butyrylcholinesterase inhibition}

Analogs $3 \mathrm{a}-3 \mathrm{q}$ showed acetylcholinesterase inhibition with $\mathrm{IC}_{50}$ values ranging between $72.04 \pm 1.12$ to $1320.65 \pm 0.95 \mu \mathrm{M}$ as compared with standard eserine $\left(\mathrm{IC}_{50}=0.85 \pm 0.0001 \mu \mathrm{M}\right)$. Only two analogs $3 \mathrm{k}$ and 30 exhibited moderate acetylcholinesterase inhibitor potential with $\mathrm{IC}_{50}$ values $72.04 \pm 1.12$ and $94.06 \pm 1.17 \mu \mathrm{M}$ respectively. Analog $3 \mathrm{k}$ was found the most potent among the series. This compound has a hydroxyl and methoxy groups on one phenyl ring and one methoxy group on other phenyl ring. The presence of these hydroxyl and methoxy groups seem to play an important role in this inhibition. The hydroxyl might be involved in hydrogen bonding. Analog 3o was found to be second active among the series. This compound illustrate activity have one thiomethyl group on one phenyl ring and a methoxy group on other phenyl part. Similarly, other active analogs have either EWG or EDG on phenyl ring, whose position, nature and arrangement on phenyl ring greatly affect the inhibition.

Analogs 3a-3p also showed a variable degree of butyrylcholinesterase inhibition with $\mathrm{IC}_{50}$ values ranging between $3.04 \pm 1.48-1876.17 \pm 0.95$ $\mu \mathrm{M}$ as compared with standard eserine $\left(\mathrm{IC}_{50}=0.85 \pm 0.0001 \mu \mathrm{M}\right)$. Five analogs $3 \mathrm{~d}, 3 \mathrm{~h}, 3 \mathrm{q}, 3 \mathrm{o}$ and $3 \mathrm{l}$ exhibited good potent butyrylcholinesterase inhibitory potential with $\mathrm{IC}_{50}$ values $3.04 \pm 1.48,9.01 \pm 0.58,15.12 \pm$ $0.66,45.00 \pm 0.99$ and $50.19 \pm 0.62 \mu \mathrm{M}$ respectively. Compound $3 \mathrm{~d}$ was found to be the most potent among the series. This compound has two chloride groups on one phenyl ring and one amino group on other phenyl ring. The presence of these two chloride group seems to be play an important role in this inhibition. The amino group might be involved in hydrogen bonding. Analog $3 \mathrm{~h}$ was found to be second most active among the series. This compound has hydroxyl moiety on one and amino group on other phenyl ring both of which might be involved in hydrogen bonding. Analog 3q has two amino groups one on each phenyl ring which might be involved in hydrogen bonding. Analog 3o has thiomethyl group on one phenyl part and methoxy group on the other phenyl part both of which are electron donating in nature. Analog $5 \mathrm{c}$ on the other hand has one electron withdrawing nitro group on one phenyl ring and methoxy group on other phenyl part. All other analogs also showed moderate to less activities.

\section{Molecular docking}

From the docking simulation and in vitro studies it was observed that compound $3 \mathrm{~d}$ (IC50: $3.00 \pm 2 \mathrm{~F} .728 \mu \mathrm{M}$ ), is the most active analog among all the compounds which are included in this study. This compound fits intensely into the active site of Butyrylcholinesterase enzyme making interactions with the residues Trp82, Gly116, Thr120, Tyr128 and Tyr332 as show in Figure 1. Trp82 was found in making hydrogen bond interaction with the hydrogen of amide NH, Gly116 established interaction with oxygen of carbonyl group, Thr120 was found in making two interactions one with carbonyl oxygen and other with the hydrogen of amide $\mathrm{NH}$, Tyr128 interact with amino group by making hydrogen bond, Tyr332 was found in arene-arene interactions with 2,4-dichlorosubstituted phenyl ring. The highest activity of compound $3 \mathrm{~d}$ might be due to the presence of two chlorine atoms attached to the same benzene ring. The chlorine atoms are well-built activating groups which polarize the molecule and facilitate it to make several interactions with other residues.

Compound $3 \mathrm{~h}$ is ranked as second most active compound establishing five interactions with different moiety of the compound as show in Figure 2, Trp82 establish $\pi$-interaction with the 2-amino

\begin{tabular}{|c|c|c|c|c|}
\hline Comp. & $\mathbf{R}_{1}$ & $\mathbf{R}_{2}$ & $\begin{array}{c}\mathrm{IC}_{50} \pm \mathrm{SEM}^{\mathrm{a}}(\mu \mathrm{M}) \\
\text { Buterycholinesterase }\end{array}$ & $\begin{array}{c}\mathrm{IC}_{50} \pm \mathrm{SEM}^{\mathrm{a}}(\mu \mathrm{M}) \\
\text { Acetylcholinesterase }\end{array}$ \\
\hline $3 a$ & & & $1220.78 \pm 1.73$ & $435.37 \pm 1.21$ \\
\hline $3 b$ & & & $250.23 \pm 0.65$ & $202.14 \pm 0.56$ \\
\hline $3 c$ & & & $135.52 \pm 1.12$ & $1320.65 \pm 0.95$ \\
\hline $3 d$ & & & $03.04 \pm 1.48$ & $394.33 \pm 0.83$ \\
\hline
\end{tabular}


Citation: Ali A, Rahman AU, Ateeq M, Ullah F, Mughal UR, et al. (2016) Conventional Versus Microwave Assisted Synthesis, Molecular Docking Studies and In Vitro Evaluation of Benzohydrazide Derivatives as New Acetylcholinesterase and Butyrylcholinesterase Inhibitors. Med Chem (Los Angeles) 6: 583-592. doi:10.4172/2161-0444.1000403

\begin{tabular}{|c|c|c|c|c|}
\hline Comp. & $\mathrm{R}_{1}$ & $\mathbf{R}_{2}$ & $\begin{array}{c}\mathrm{IC}_{50} \pm \mathrm{SEM}^{\mathrm{a}}(\mu \mathrm{M}) \\
\text { Buterycholinesterase }\end{array}$ & $\begin{array}{c}\mathrm{IC}_{50} \pm \mathrm{SEM}^{\mathrm{a}}(\mu \mathrm{M}) \\
\text { Acetylcholinesterase }\end{array}$ \\
\hline $3 e$ & & & $1876.17 \pm 0.95$ & $243.36 \pm 0.90$ \\
\hline $3 f$ & & & $316.59 \pm 1.50$ & $560.47 \pm 0.77$ \\
\hline $3 g$ & & & -- & -- \\
\hline $3 \mathrm{~h}$ & & & $09.01 \pm 0.58$ & $380.21 \pm 0.58$ \\
\hline $3 i$ & & & $1300.65 \pm 1.20$ & $N / A$ \\
\hline $3 \mathrm{j}$ & & & $243.21 \pm 0.21$ & $250.54 \pm 0.56$ \\
\hline $3 \mathrm{k}$ & & & $188.87 \pm 1.32$ & $72.04 \pm 1.12$ \\
\hline 31 & & & $50.19 \pm 0.62$ & $117.13 \pm 1.16$ \\
\hline $3 m$ & & & $1408.69 \pm 1.76$ & $375.61 \pm 0.84$ \\
\hline $3 n$ & & & $1560.45 \pm 0.95$ & $402.25 \pm 1.25$ \\
\hline
\end{tabular}


Citation: Ali A, Rahman AU, Ateeq M, Ullah F, Mughal UR, et al. (2016) Conventional Versus Microwave Assisted Synthesis, Molecular Docking Studies and In Vitro Evaluation of Benzohydrazide Derivatives as New Acetylcholinesterase and Butyrylcholinesterase Inhibitors. Med Chem (Los Angeles) 6: 583-592. doi:10.4172/2161-0444.1000403

\begin{tabular}{|c|c|c|c|c|}
\hline Comp. & $\mathbf{R}_{1}$ & $\mathbf{R}_{2}$ & $\begin{array}{c}\mathrm{IC}_{50} \pm \mathrm{SEM}^{\mathrm{a}}(\mu \mathrm{M}) \\
\text { Buterycholinesterase }\end{array}$ & $\begin{array}{c}\mathrm{IC}_{50} \pm \mathrm{SEM}^{\mathrm{a}}(\mu \mathrm{M}) \\
\text { Acetylcholinesterase }\end{array}$ \\
\hline 30 & & & $45.00 \pm 0.99$ & $94.06 \pm 1.17$ \\
\hline $3 p$ & & & $168.11 \pm 1.81$ & $362.81 \pm 0.50$ \\
\hline $3 q$ & & & $15.12 \pm 0.66$ & $335.93 \pm 1.01$ \\
\hline eserine & & & $0.85 \pm 0.0001$ & $0.85 \pm 0.0001$ \\
\hline
\end{tabular}

Table 1: Buterycholinesterase and Acetylcholinesterase Inhibition by Benzohydrazide Derivatives.

\begin{tabular}{|c|c|c|c|c|c|}
\hline \multirow{2}{*}{ Comp. } & \multirow{2}{*}{ m. p. $\left({ }^{\circ} \mathrm{C}\right)$} & \multicolumn{2}{|c|}{ Conventional } & \multicolumn{2}{|c|}{ MW Irradiation } \\
\hline & & Time (min) & Yield (\%) & Time (min) & Yield (\%) \\
\hline $3 a$ & 274 & 165 & 82 & 5 & 84 \\
\hline $3 b$ & 138 & 240 & 79 & 5 & 80 \\
\hline $3 c$ & 243 & 120 & 84 & 3 & 83 \\
\hline $3 d$ & 209 & 150 & 86 & 4 & 89 \\
\hline $3 e$ & 234 & 210 & 83 & 5 & 81 \\
\hline $3 f$ & 231 & 240 & 82 & 5 & 85 \\
\hline $3 g$ & 125 & 240 & 85 & 5 & 85 \\
\hline $3 \mathrm{~h}$ & 218 & 180 & 78 & 4 & 81 \\
\hline $3 \mathrm{i}$ & 147 & 150 & 82 & 5 & 88 \\
\hline $3 j$ & 281 & 130 & 84 & 5 & 88 \\
\hline $3 k$ & 117 & 180 & 83 & 4 & 84 \\
\hline 31 & 212 & 90 & 85 & 4 & 85 \\
\hline $3 m$ & 184 & 150 & 80 & 5 & 81 \\
\hline $3 n$ & 130 & 180 & 84 & 3 & 82 \\
\hline 30 & 172 & 180 & 84 & 5 & 85 \\
\hline $3 p$ & 187 & 180 & 86 & 5 & 91 \\
\hline $3 q$ & 253 & 120 & 84 & 5 & 83 \\
\hline
\end{tabular}

Table 2: Convential vs Microwave Assisted Synthesis of Benzohydrazide Derivatives

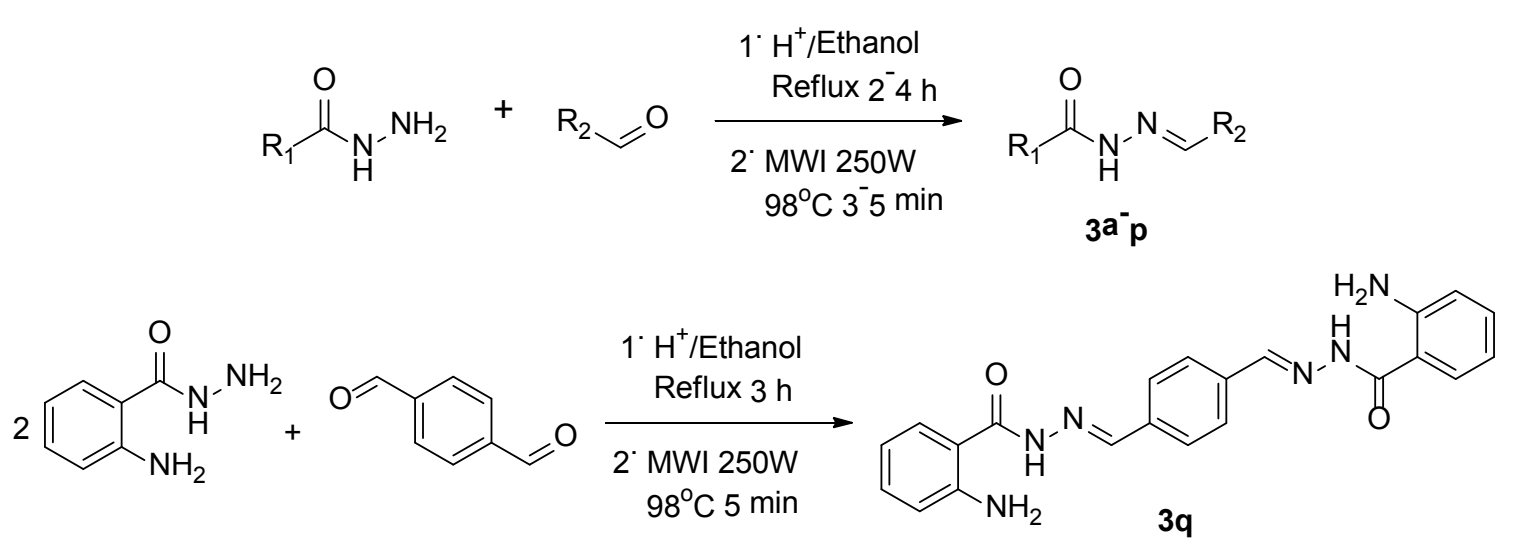

Special case

Scheme 1: Synthesis of Bezohydrazide Derivatives. 
Citation: Ali A, Rahman AU, Ateeq M, Ullah F, Mughal UR, et al. (2016) Conventional Versus Microwave Assisted Synthesis, Molecular Docking Studies and In Vitro Evaluation of Benzohydrazide Derivatives as New Acetylcholinesterase and Butyrylcholinesterase Inhibitors. Med Chem (Los Angeles) 6: 583-592. doi:10.4172/2161-0444.1000403

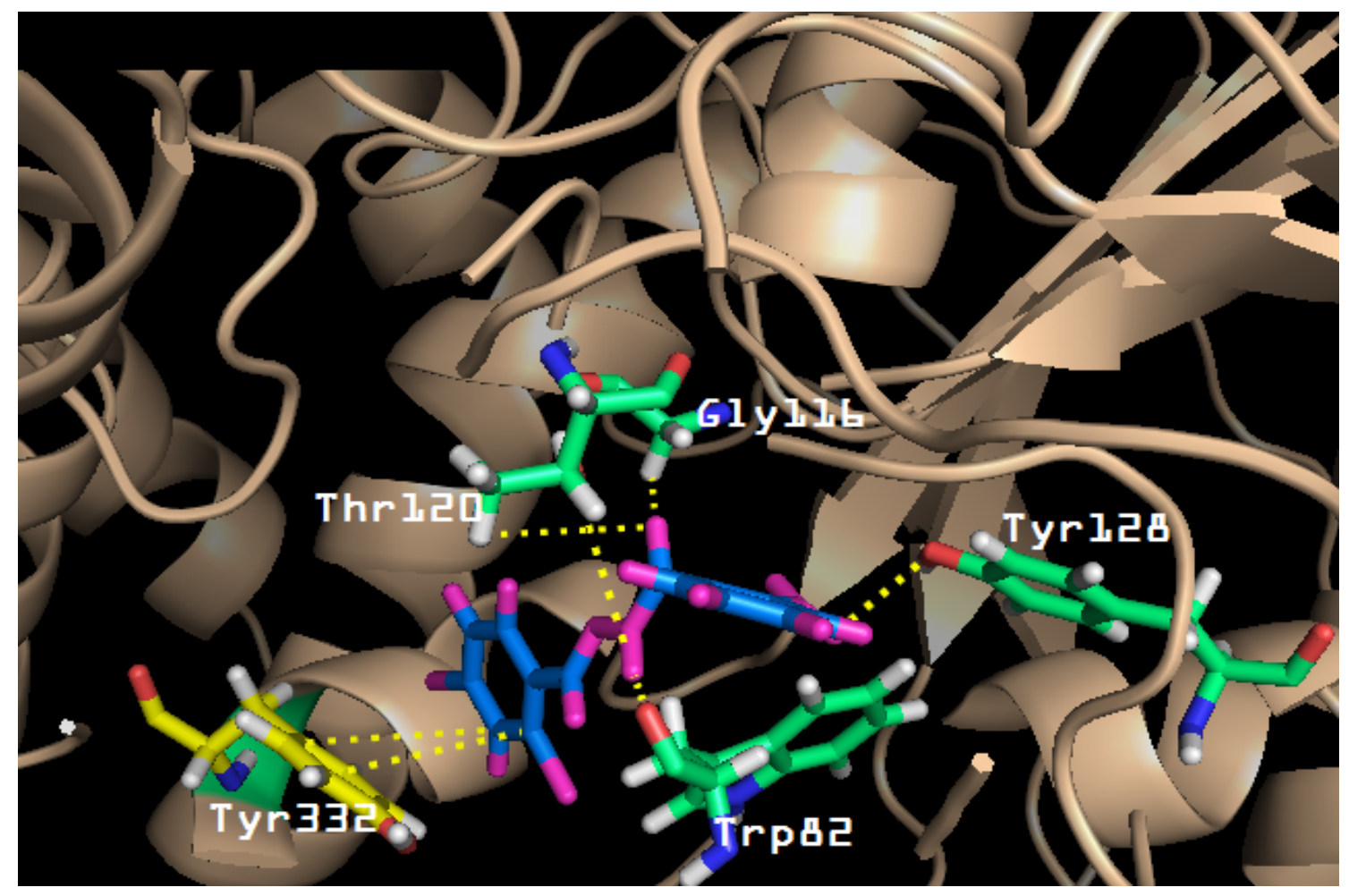

Figure 1: 3D Interactions of Butyrylcholinesterase Enzyme with Compound 3d.

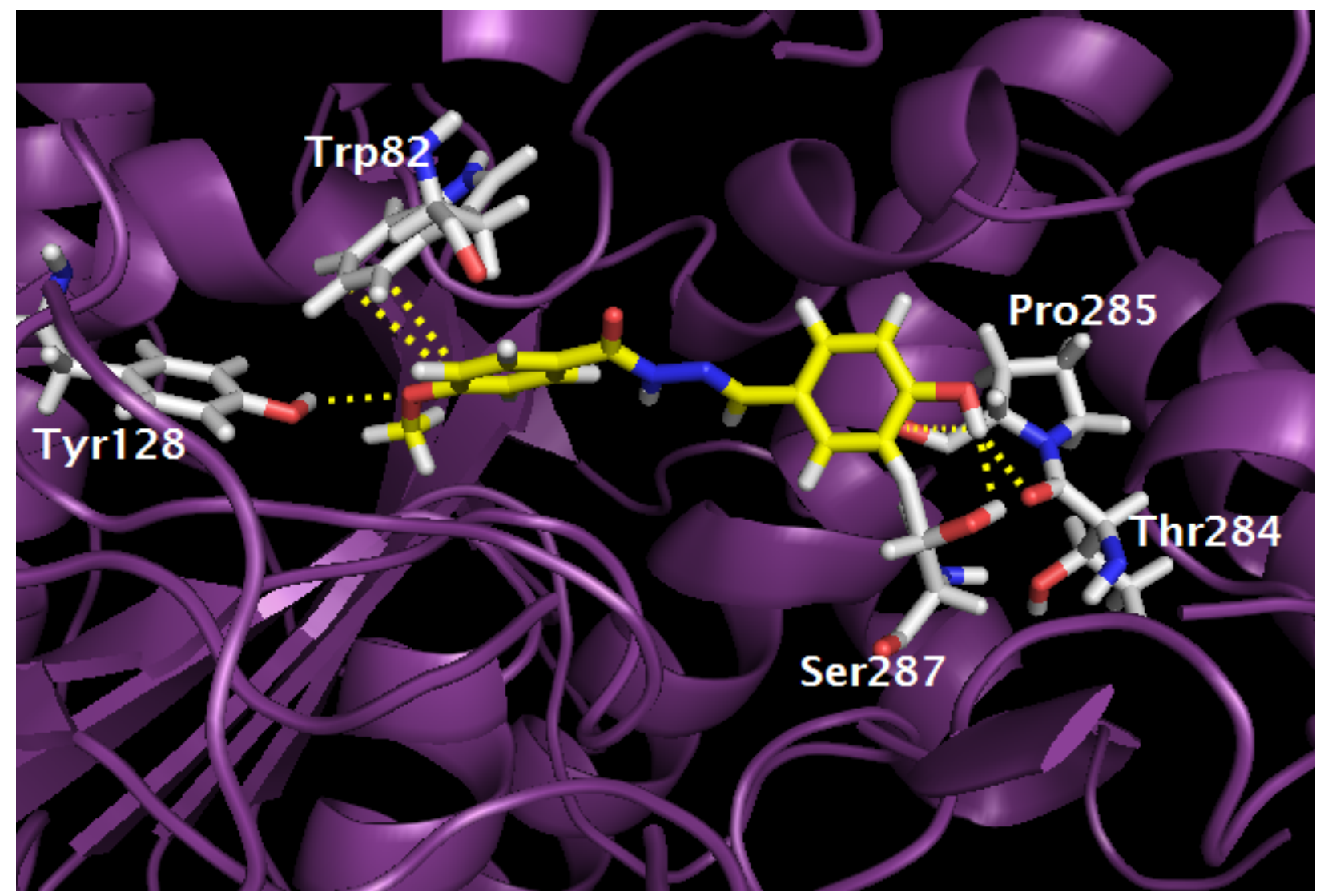

Figure 2: 3D Interactions of Butyrylcholinesterase Enzyme with Compound 3h. 
Citation: Ali A, Rahman AU, Ateeq M, Ullah F, Mughal UR, et al. (2016) Conventional Versus Microwave Assisted Synthesis, Molecular Docking Studies and In Vitro Evaluation of Benzohydrazide Derivatives as New Acetylcholinesterase and Butyrylcholinesterase Inhibitors. Med Chem (Los Angeles) 6: 583-592. doi:10.4172/2161-0444.1000403

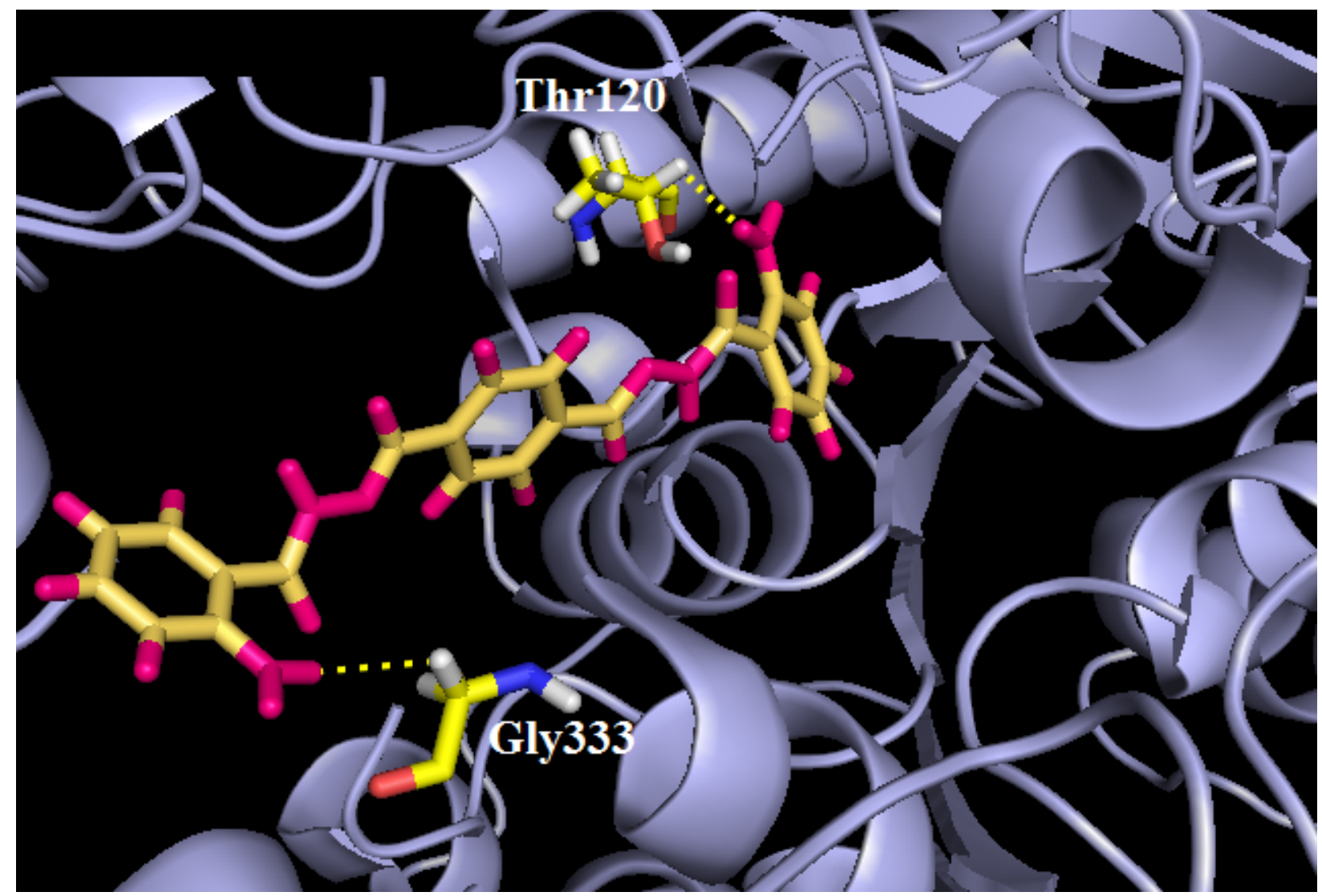

Figure 3: 3D Interactions of Butyrylcholinesterase Enzyme with Compound 3q.

substituted benzene ring. Glu197 and Pro285 make hydrogen bond interactions with the hydrogen of amino and $\mathrm{OH}$ groups attached on two different benzene rings respectively. Tyr332 establish $\pi$-interaction with the 4-hydroxy substituted benzene ring. His438 interact with the hydrogen of amino through hydrogen bond.

Compound $3 \mathrm{q}\left(\mathrm{IC}_{50}=15.00 \pm 2.728 \mu \mathrm{M}\right)$ with two binding interactions is rank third moderate active compound. The hydrogen of amino group on each benzene ring of the compound establishes hydrogen interactions with Thr120 and Gly133 as show in Figure 3. Compounds $3 \mathrm{j}$ and $3 \mathrm{c}$ also exhibited weak potency against $\mathrm{BChE}$.

The rest of the compounds reflects no interaction with the active site residues of $\mathrm{BChE}$, which might be due to the inverted orientation, and these compounds also show least inhibitory activity (IC50 value) against the human BChE enzyme which contain much differences, if compared with the standard substrate (Eserine: IC50 value $0.85 \pm$ 0.0001). Therefore, these compounds were not further evaluated for further study.

The structural difference between the most active compound $3 \mathrm{n}$ and the least active compounds are the Ortho and Para-oriented chlorine atoms of the compound. These chlorine moieties may provide enzymatic potency against butyrylcholinesterase enzyme.

Docking results of compound $3 \mathrm{~d}, 3 \mathrm{~h}$ and $3 \mathrm{q}$ with $\mathrm{BChE}$ provided valuable information about the nature of the binding interactions that were delightfully associated with the experimental studies. This information could be utilized to design new leads against the BChE.
The molecular docking study of these compounds revealed that the ligands with polar, light and nucleophilic groups like chlorine atom and hydroxyl group showed better interaction mode and high docking score against the target protein and therefore have good inhibitory activities. On the other hands ligands with electrophilic group like nitro group, non-polar like methyl or benzene and bulky groups have shown poor interactions and low docking scores.

\section{Computational methods}

The current study was designed to dock Schiff base derivatives (codes)in the active site of Butyrylcholinesterase (BChE) enzyme with the following PC specifications; Intel ${ }^{(\mathrm{R})} \mathrm{xenon}^{(\mathrm{R})} \mathrm{CPU}$ E5620@2.40 GHz system having 3.8 GB RAM with the open 11.4 (X 86_64) operating platform. Protein-Ligand docking was carried out using the Molecular Operating Environment (MOE 2010.11) software package. Among thirty-one X-ray crystal structures of human $\mathrm{BChE}$ in the protein data bank [33,34] (Pdb code 1P0P with $2.30 \AA$ resolution) was selected as the target protein based on suitable resolution and co-crystallized Ligand, BCh. The entire system (target protein) was energy minimized by MMFF94x force field [35], after adding the missing hydrogen atoms. 3D structures of all seventeen synthesized compounds were built by molecule builder which is incorporated in MOE modeling package and the structures (Ligands) were subjected to MMFF94x for energy minimization.

Subsequently for the evaluation of potential energy, partial charges were calculated by MMFF94x force field [36]. Both prepared systems (protein and Ligand) were introduced for molecular docking simulation. Docking simulations were performed by using Triangle 
Citation: Ali A, Rahman AU, Ateeq M, Ullah F, Mughal UR, et al. (2016) Conventional Versus Microwave Assisted Synthesis, Molecular Docking Studies and In Vitro Evaluation of Benzohydrazide Derivatives as New Acetylcholinesterase and Butyrylcholinesterase Inhibitors. Med Chem (Los Angeles) 6: 583-592. doi:10.4172/2161-0444.1000403

matcher placement method. A total of 30 conformations were generated for each Ligand protein complex with docking score. Each complex was analyzed for interactions and their 3D images were taken.

The default parameters of MOE-Dock program were used for the molecular docking of the ligands. At the end of docking, the best conformations were analyzed for hydrogen bonding $/ \pi-\pi$ interactions.

\section{Anticholestenerase assays}

AChE from electric eel and BChE from equine serum were used to explore the enzyme inhibitory potential of benzohydrazide derivatives, using Ellman's assay [37,38]. The assay is based on the hydrolysis of acetlthiocholine iodide butylrathiocholestine iodide by respective enzymes and the formation of 5-thio-2-nitrobenzoate anion followed by complexation with DNTB to give yellow color compound which is detected by spectrophotometer beside reaction.

Preparation of solutions: Benzohydrazide derivatives were dissolved in phosphate buffer $(0.1 \mathrm{M})$ in concentration ranging from $125-1000 \mu \mathrm{g} / \mathrm{ml}$. for preparation of $0.1 \mathrm{M}$ and $8.0 \pm 0.1 \mathrm{pH}$ phosphate buffer solution $\mathrm{K}_{2} \mathrm{HPO}_{4}$ and $\mathrm{KH}_{2} \mathrm{PO}_{4}(13 \mathrm{~g} / \mathrm{L})$ were prepared and were mixed in $94 \%$ and $6 \%$ ratio respectively. Finally, KOH was added to adjust the $\mathrm{pH}$. $\mathrm{BChE}(7-16 \mathrm{U} / \mathrm{ml})$ was diluted in freshly prepared buffer solution until final concentrations of $0.03 \mu / \mathrm{ml}$ and $0.01 \mu / \mathrm{ml}$ were obtained. Solutions of DNTB $(0.0002273 \mathrm{M})$, ATchI $(0.0005 \mathrm{M})$ were prepared in distilled water and were kept in eppendorf caps in refrigerator at $8^{\circ} \mathrm{C}$. Galanthamine (Positive control) was dissolved in methanol and aforementioned dilutions were prepared.

Spectroscopic analysis: For each assay, an enzyme solution of 5 $\mu \mathrm{l}$ was added to the cuvette followed by addition of samples solution $(205 \mu \mathrm{l})$, and finally DTNB reagent $(5 \mu \mathrm{l})$. The solution mixture was maintained at $30^{\circ} \mathrm{C}$ for 15 min using water bath with subsequent addition of substrate solution $(5 \mu \mathrm{l})$ was added. A double beam spectrophotometer (Thermo electron corporation USA) was used to measure the absorbance at $412 \mathrm{~nm}$. Negative control contained all components apart from the sample solutions, whereas positive control eserine $(10 \mu \mathrm{g} / \mathrm{ml})$ was used in the assay as standard cholinesterase inhibitor. The absorbance along with the reaction time were taken for four minutes at $30^{\circ} \mathrm{C}$. The experiments were performed in triplicate. The enzyme activity and enzyme inhibition by control and tested samples were calculated from the rate of absorption with change in time

\section{$(\mathrm{V}=\Delta \mathrm{Abs} / \Delta \mathrm{t})$ as follow;}

Enzyme inhibition (\%)=100-percent enzyme activity

Enzyme activity $(\%)=100 \times \mathrm{V} / \mathrm{V}_{\max }$

where $\left(\mathrm{V}_{\max }\right)$ is enzyme activity in the absence of inhibitor drug

Statistical data analysis: Anticholestenerase inhibitory ability was calculated by a linear regression analysis among the percent inhibition against the samples concentrations via Microsoft Excel 365 program. All the assays were repeated in triplicate and vales were expressed as means \pm S.E.M. $\mathrm{IC}_{50}$ values were calculated using EZ-Fit Enzyme kinetics software (Perrella Scientific Inc. Amherst, USA).

\section{Conclusion}

Seventeen analogs were prepared through conversational as well as microwave irradiation and evaluated for acetylcholinesterase and butyrylcholinesterase inhibition. Only two analogs $3 \mathrm{k}$ and $3 \mathrm{o}$ exhibited moderate acetylcholinesterase inhibitor potential with $\mathrm{IC}_{50}$ values $72.04 \pm 1.12$ and $94.06 \pm 1.17 \mu \mathrm{M}$ respectively, compared to the standard eserine $\left(\mathrm{IC}_{50}=0.85 \pm 0.0001 \mu \mathrm{M}\right)$. Five analogs $3 \mathrm{~d}, 3 \mathrm{~h}, 3 \mathrm{q}, 3 \mathrm{o}$ and $3 l$ exhibited good potent butyrylcholinesterase inhibitory potential with $\mathrm{IC}_{50}$ values $03.04 \pm 1.48,09.01 \pm 0.58,15.12 \pm 0.66,45.00 \pm 0.99$ and $50.19 \pm 0.62 \mu \mathrm{M}$ respectively. All other analogs exhibited moderate to less enzyme inhibition. The binding affinity was confirmed through molecular docking studies. All compounds were characterized through FTIR, ${ }^{1} \mathrm{H}$ NMR, ${ }^{13} \mathrm{C}$ NMR and EI-MS.

\section{Acknowledgements}

This work was financially supported by Higher Education Commission, Pakistan

\section{References}

1. Mingos DMP, Whittaker AG (1997) Microwave dielectric heating effects in chemical synthesis. John Wiley and Sons, and Spectrum Akademischer Verlag: New York, Heidelberg, USA, pp: 479-514.

2. Thostenson ET, Chou TW (1999) Microwave processing: fundamentals and applications. Composites Part A: Applied Science and Manufacturing 30: 1055-1071.

3. Xu Y, Guo QX (2004) Syntheses of heterocyclic compounds under microwave irradiation. Heterocycles 63: 903-974.

4. Langa F, De la Cruz P, Espildora E, De la Hoz A (2000) Applications of Microwave Irradiation to Fullerene Chemistry. Fullerenes, The Electrochemical Society, New York 9: 168-178.

5. de la Hoz A, Díaz-Ortis A, Moreno A, Langa F (2000) Cycloadditions under microwave irradiation conditions: Methods and applications. European Journal of Organic Chemistry 2000: 3659-3673

6. Zong L, Zhou S, Sgriccia N, Hawley MC, Kempel LC (2002) A review of microwave-assisted polymer chemistry (MAPC). The Journal of microwave power and electromagnetic energy: a publication of the International Microwave Power Institute 38: 49-74.

7. Larhed M, Moberg C, Hallberg A (2002) Microwave-accelerated homogeneous catalysis in organic chemistry. Accounts of chemical research 35: 717-727.

8. Will H, Scholz P, Ondruschka B (2002) Heterogene gasphasenkatalyse im mikrowellenfeld. Chemie Ingenieur Technik 74: 1057-1067.

9. Bose AK, Manhas MS, Ganguly SN, Sharma AH, Banik BK (2002) MORE chemistry for less pollution: Applications for process development. Synthesis 2002: 1578-1591.

10. Anand R, Gill KD, Mahdi AA (2014) Therapeutics of Alzheimer's disease: Past present and future. Neuropharmacology 76: 27-50.

11. Korolev IO (2014) Alzheimer's Disease: A Clinical and Basic Science Review. Medical Student Research Journal 4: 24-33.

12. De la Torre JC (2002) Alzheimer disease as a vascular disorder nosological evidence. Stroke 33: 1152-1162.

13. Ott A, Breteler MM, Van Harskamp F, Claus JJ, Van Der Cammen TJ, et al. (1995) Prevalence of Alzheimer's disease and vascular dementia: association with education. The Rotterdam study, Bmj 310: 970-973.

14. Wimo, M. Prince (2010) World Alzheimer Report 2010: The Global Economic Impact of Dementia. Alzheimer's disease International.

15. Cavalli A, Bolognesi ML, Minarini A, Rosini M, Tumiatti V, et al. (2008) Multitarget-directed ligands to combat neurodegenerative diseases. Journal of medicinal chemistry $51: 347-372$

16. Giacobini E (2003) Cholinesterases: new roles in brain function and in Alzheimer's disease. Neurochemical research 28: 515-522.

17. Holzgrabe U, Kapková P, Alptüzün V, Scheiber J, Kugelmann E (2007) Targeting acetylcholinesterase to treat neurodegeneration. Expert opinion on therapeutic targets 11: 161-179.

18. Greig NH, Lahiri DK, Sambamurti K (2002) Butyrylcholinesterase: an important new target in Alzheimer's disease therapy. International Psychogeriatrics 14: 77-91.

19. Masson P, Lockridge $O$ (2010) Butyrylcholinesterase for protection from organophosphorus poisons: catalytic complexities and hysteretic behavior Archives of biochemistry and biophysics 494: 107-120. 
Citation: Ali A, Rahman AU, Ateeq M, Ullah F, Mughal UR, et al. (2016) Conventional Versus Microwave Assisted Synthesis, Molecular Docking Studies and In Vitro Evaluation of Benzohydrazide Derivatives as New Acetylcholinesterase and Butyrylcholinesterase Inhibitors. Med Chem (Los Angeles) 6: 583-592. doi:10.4172/2161-0444.1000403

20. Sridhar SK, Saravanan M, Ramesh A (2001) Synthesis and antibacterial screening of hydrazones, Schiff and Mannich bases of isatin derivatives. European Journal of Medicinal Chemistry 36: 615-625.

21. Bharti SK, Nath G, Tilak R, Singh SK (2010) Synthesis, anti-bacterial and anti-fungal activities of some novel Schiff bases containing 2, 4-disubstituted thiazole ring. European journal of medicinal chemistry 45: 651-660.

22. da Silva CM, da Silva DL, Modolo LV, Alves RB, de Resende MA, et al. (2011) Schiff bases: a short review of their antimicrobial activities. Journal of Advanced research 2: 1-8.

23. Kumar KS, Ganguly S, Veerasamy R, De Clercq E (2010) Synthesis, antivira activity and cytotoxicity evaluation of Schiff bases of some 2-phenyl quinazoline-4 (3) H-ones. European Journal of Medicinal Chemistry 45: 5474-5479.

24. Hranjec M, Starčević K, Pavelić SK, Lučin P, Zamola GK, et al. (2011) Synthesis, spectroscopic characterization and antiproliferative evaluation in vitro of novel Schiff bases related to benzimidazoles. European journal of medicinal chemistry 46: 2274-2279.

25. Tarafder MT, Kasbollah A, Saravanan N, Crouse KA, et al. (2002) S-methyldithiocarbazate and its Schiff bases: evaluation of bondings and biological properties. Journal of biochemistry, molecular biology, and biophysics: JBMBB: the official journal of the Federation of Asian and Oceanian Biochemists and Molecular Biologists (FAOBMB) 6: 85-91.

26. Li YF, Liu ZQ (2011) Ferrocenyl Schiff base as novel antioxidant to protect DNA against the oxidation damage. European Journal of Pharmaceutical Sciences, 44: 158-163.

27. Bhat MA, Al-Omar MA (2011) Synthesis, characterization and in vivo anticonvulsant and neurotoxicity screening of Schiff bases of phthalimide. Acta poloniae pharmaceutica 68: 375-380.

28. El-Sayed NA, Awadallah FM, Ibrahim NA, El-Saadi MT (2010) Synthesis, antiinflammatory and ulcerogenicity studies of some substituted pyrimido [1, 6-a] azepine derivatives. European journal of medicinal chemistry 45: 3147-3154.

29. Kasabe A, Mohite V, Ghodake J, Vidhate J (2010) Synthesis, Characterization and Primary Antimicrobial, Antifungal Activity Evaluation of Schiff bases of
4-Chloro-(3-substituted-phenylimino)-methyl-[2H]-chromene-2-one. Journal of Chemistry $7: 377-382$.

30. Pandeya SN, Sriram D, Nath G, DeClercq E (1999) Synthesis, antibacterial, antifungal and anti-HIV activities of Schiff and Mannich bases derived from isatin derivatives and $\mathrm{N}$-[4-(4'-chlorophenyl) thiazol-2-yl] thiosemicarbazide. European Journal of Pharmaceutical Sciences 9: 25-31.

31. Khan KM, Khan M, Ali M, Taha M, Rasheed S, et al. (2009) Synthesis of bisSchiff bases of isatins and their antiglycation activity. Bioorganic \& medicinal chemistry 17: 7795-7801.

32. Sashidhara KV, Rosaiah JN, Bhatia G, Saxena JK (2008) Novel keto-enamine Schiffs bases from 7-hydroxy-4-methyl-2-oxo-2H-benzo [h] chromene-8, 10-dicarbaldehyde as potential antidyslipidemic and antioxidant agents. European journal of medicinal chemistry 43: 2592-2596.

33. Berman HM, Westbrook J, Feng Z, Gilliland G, Bhat TN, et al. (2000) The protein data bank. Nucleic Acids Res 28: 235-242.

34. Nicolet $Y$, Lockridge O, Masson P, Fontecilla-Camps JC, Nachon F (2003) Crystal structure of human butyrylcholinesterase and of its complexes with substrate and products. J Biol Chem 278: 41141-41147.

35. Halgren TA (1999) MMFF VI. MMFF94s option for energy minimization studies. J Comput Chem 20: 720-729.

36. Halgren TA (1996) Merck molecular force field. I. Basis, form, scope parameterization, and performance of MMFF94. J Comput Chem 17: 490-519.

37. Ellman GL, Courtney KD, Andres V, Featherstone RM (1961) A new and rapid colorimetric determination of acetylcholinesterase activity. Biochem. Pharmacol 7: 88-95.

38. Maeda H, Matsuno H, Ushida M, Katayama K, Saeki K, et al. (2005) 2, 4-Dinitrobenzenesulfonyl Fluoresceins as Fluorescent Alternatives to Ellman's Reagent in Thiol-Quantification Enzyme Assays. Angewandte Chemie 117 2982-2985. 